\title{
The Man With the Swollen Tongue
}

\author{
Celia Maia Cruz ${ }^{\mathrm{a}, \mathrm{b}, \mathrm{d}}$, Joao Oliveira ${ }^{\mathrm{a}}$, Teresa Sequeira ${ }^{\mathrm{a}}$, Miguel Ricardo ${ }^{\mathrm{a}}$,, Luis Magalhaes ${ }^{\mathrm{c}}$, \\ Catarina Mendonca ${ }^{\mathrm{a}, \mathrm{b}}$
}

\begin{abstract}
Eosinophilia is defined as an elevation of the eosinophil count above $0.5 \times 10^{9} / \mathrm{L}$ and it can be further classified in mild, moderate or severe according to blood eosinophil count. It is important to recognize the combination of eosinophilia and symptoms caused by eosinophils in order to make the correct diagnostic workup, so that distinction between secondary, primary and idiopathic forms can be made. The authors present a case of a 72-year-old man with asthma diagnosed during adulthood. He had a history of diarrhea for the last 9 months already investigated with abdominal imaging and colonoscopy and interpreted as a pancolitis of undetermined etiology. He presented to the emergency department complaining of a swollen tongue in the last 2 weeks. His laboratory studies showed leukocytosis and eosinophilia with a negative HIV antibody test. CT scan of the head and neck revealed diffuse thickening of the intrinsic muscles of the tongue. As for the chest CT, it showed right and left upper lobe patchy opacities. Biopsies of the regions affected (tongue, small intestine and colon) showed abundant infiltration by polymorphonuclear eosinophils. The cytoplasmic anti-neutrophil cytoplasmic antibody (C-ANCA) was elevated and, according to the clinical and laboratory data described before, the patient was diagnosed with Churg-Strauss syndrome (CSS). Treatment with corticosteroids and cyclophosphamide led to complete remission of symptoms and eosinophilia. Clinicians must be aware of eosinophil-associated disease, an entity that refers to a wide spectrum of disorders but in which eosinophils play a central role. Eosinophilic granulomatosis with polyangiitis (EGPA), formerly known as CSS, is a rare systemic vasculitis and a better understanding of the disease is still needed.
\end{abstract}

Keywords: Eosinophilia; Churg-Strauss syndrome; Vasculitis

\footnotetext{
Manuscript accepted for publication October 17, 2016

aDepartment of Internal Medicine, Hospital de Santo Antonio, Centro Hospitalar do Porto, Portugal

' Instituto de Ciencias Biomedicas Abel Salazar, Universidade do Porto, Portugal

'Department of Internal Medicine, Hospital Luz/Arrabida, Porto, Portugal ${ }^{\mathrm{d} C}$ Corresponding Author: Celia Maia Cruz, Department of Internal Medicine, Hospital de Santo Antonio, Centro Hospitalar do Porto, Largo Prof. Abel Salazar, 4099-001 Porto, Portugal. Email: celiamaiacruz@gmail.com
}

doi: https://doi.org/10.14740/jmc2677w

\section{Introduction}

Paul Ehrlich first described the eosinophil in 1879 and named it after Eos, the Greek goddess of the dawn. Much has been learned about the physiology and function of eosinophils ever since and it is currently known that they produce a large panel of secreted cytokines and soluble mediators and of bidirectional interactions with dendritic and $\mathrm{T}$ cells, thus being involved in initiation and propagation of diverse inflammatory responses, as well as modulating innate and adaptive immunity $[1,2]$.

Eosinophilia is defined as a blood eosinophil count above the upper limit of reference (in adults $\geq 0.5 \times 10^{9} / \mathrm{L}$ ) and can be classified into mild $\left(0.5-1.5 \times 10^{9} / \mathrm{L}\right)$, moderate $(1.5-5.0$ $\left.\times 10^{9} / \mathrm{L}\right)$ or severe $\left(>5.0 \times 10^{9} / \mathrm{L}\right)$. Eosinophilia can also be divided into three different categories: reactive (or secondary), clonal (or primary) and idiopathic hypereosinophilic syndrome (HES), the last being defined by moderate or severe eosinophilia of unknown origin, persisting more than 6 months with organ damage.

The most common cause of eosinophilia is allergy in the western world and invasive parasite infections in the developing countries and damage to end-organs can result even in eosinophilia secondary to a known cause as a Toxocara infection [3]. End-organ damage is caused by activation of eosinophils, release of their granules contents and deposition of their proteins and thrombotic events [4]. Treatment, therefore, must rely in destroy or suppress eosinophils and, for that reason, clinicians must be aware of eosinophil-associated disease which can manifest in a wide variety of manners.

Eosinophilic granulomatosis with polyangiitis (EGPA) is a rare cause of eosinophilia and its prevalence (10 - 15/million) and annual incidence (0.9-1.2/million) are about 10 times lower than those of other anti-neutrophil cytoplasmic antibody (ANCA)-associated vasculitis such as granulomatosis with polyangiitis (GPA) and microscopic polyangiitis (MPA) [5]. The role of ANCAs in EGPA, detectable in only $30-40 \%$ of patients, remains unclear and eosinophils, which play no role in GPA or MPA, seem to be important in EGPA [5-7].

\section{Case Report}

A 72-year-old man, with a history of asthma diagnosed in the adult life, was admitted to our tertiary care hospital complain- 

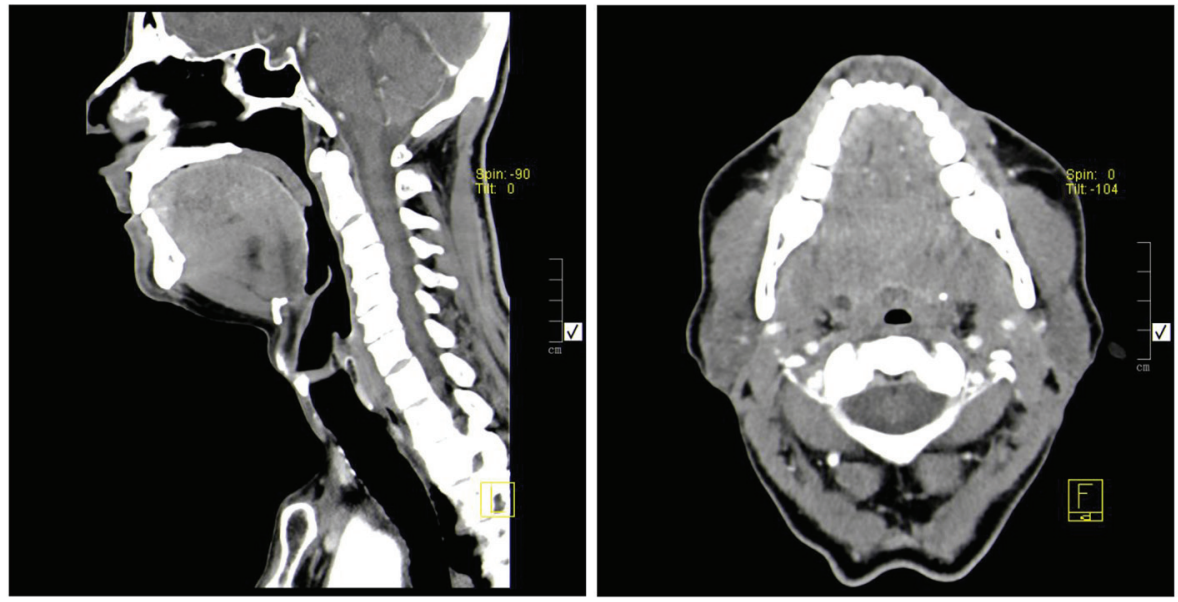

Figure 1. Diffuse thickening of the intrinsic muscles of the tongue, without contrast enhancement changes. No changes consistent with nodular lesions.

ing of a swollen tongue and submental edema in the last 2 weeks. He had been well until 9 months before this presentation, when diarrhea developed, with five to six stools per day, with no blood or mucus. During that time, he lost $17 \%$ of his body weight. Five months before admission, he performed an abdominal ultrasound that showed, in the lower right abdomen, parietal thickening of the last ileal loop and small adenopathies adjacent to this region. A colonoscopy was then proposed and some scattered erosions were visible. Biopsies revealed a mononuclear inflammatory infiltrate with nodular aggregation and some polymorphonuclear eosinophils. He had at that time a complete blood count where lymphocytosis and eosinophilia were already present. The diagnosis of a pancolitis of undetermined etiology was made and the patient was started on mesalazine with a reduction in the number of stools (two per day).

However, 2 weeks before admission, he began feeling a swollen tongue and posteriorly a submental swelling, with no palpable lymph nodes, and for that reason, was admitted into the emergency department.

On examination, he was afebrile with a pulse rate of 70 beats per minute and a blood pressure of $109 / 79 \mathrm{~mm} \mathrm{Hg}$. His respiratory rate was 16 breaths per minute while breathing room air, with normal peripheral oxygen saturation. He had no nasal polyps and no cutaneous lesions. The patient had fine crackles on the inferior parts of both lungs. Abdominal examination and ultrasound were innocent. His laboratory tests showed elevated white blood count of $13.89 \times 10^{9} / \mathrm{L}\left(4.00-11.00 \times 10^{9} / \mathrm{L}\right)$, and eosinophilia of $50 \%\left(6.96 \times 10^{9} / \mathrm{L}\right)$. Electrolytes, renal function, liver enzymes and urinalysis were normal. He had normal tryptase and vitamin B12 values and a negative HIV test. Parasite infection was excluded since he had no history of recent travel and stool and blood analyses were negative. A diffuse thickening of the tongue muscles was observed by CT imaging (Fig. 1). He was then proposed for a chest CT scan that showed patchy airspace opacities in the upper lobes (Fig. 2 ). Bronchoscopy revealed a diffuse phenomenon of bronchitis with mucosal congestion with no other changes or microbiological isolations in bronchoalveolar lavage.

The medulograma and bone marrow aspirate were normocellular and the immunophenotypic study showed no changes suggestive of lymphoproliferative disease. The FIP1L1-PDGFRA mutation was also negative.
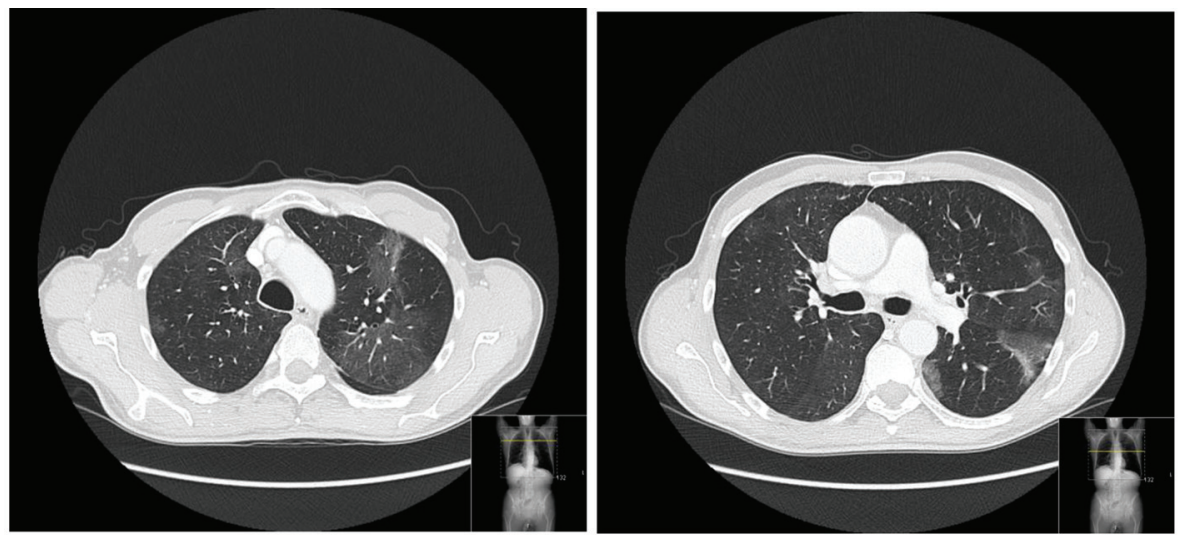

Figure 2. Lung fields with normal amplitude but discrete low density alveolar ground-glass opacities scattered by the upper lung lobes. 
The antibody profile, rheumatoid factor, anti-glomerular basement membrane antibody, perinuclear anti-neutrophil cytoplasmic antibodies (P-ANCA), myeloperoxidase antibodies (MPO) and complements were normal. Cytoplasmic ANCA (C-ANCA) (1/80) and the anti-proteinase-3 (PR3) were elevated.

Endoscopy was performed and colonoscopy was repeated (similar to the previous) and new biopsies were made that showed abundant infiltration by polymorphonuclear eosinophils, congruent with eosinophil-associated disease.

A tongue biopsy was also made and the extracted lymphoid population had no immunophenotypic alterations suggestive of lymphoma. The histopathologic study showed no dysplasia and identified a marked inflammatory infiltrate consisting of eosinophils and scant lymphocytes.

Even thought the patient had no symptoms suggestive of heart or central nervous involvement, a complete neurological examination was performed, and electrocardiogram, transthoracic echocardiogram, $\mathrm{N}$-terminal pro-brain natriuretic peptide and troponin measurements were made with normal results.

In view of these findings, the diagnosis of EGPA was made and the patient was started treatment on prednisolone (1 $\mathrm{mg} / \mathrm{kg} /$ day $)$ and cyclophosphamide $\left(0.6 \mathrm{~g} / \mathrm{m}^{2}\right)$. The patient is currently asymptomatic, with no diarrhea or swollen tissues, and a normal blood count.

\section{Discussion}

EGPA is a systemic necrotizing vasculitis that affects smallto-medium-sized vessels. In 1990, the American College of Rheumatology based the diagnosis of CSS on the presence of four of six criteria: asthma, sinusitis, pulmonary infiltrates, eosinophilia $>10 \%$ in peripheral blood, histological proof of vasculitis with extravascular eosinophils and evidence of neuropathy [8]. Our patient fulfilled four of these criteria and therefore the diagnosis was made even though no vasculitic lesions were present. In fact, EGPA may present in three distinct phases: the prodromal phase, characterized by asthma and rhinosinusitis, the eosinophilic phase, marked by peripheral eosinophilia and organ involvement and the vasculitic phase. These three phases are not necessarily seen in the same order and vasculitis may not be present at diagnosis.

The ANCA test results may help define subgroups of patients and there is evidence to suggest that ANCA-positive patients are more likely to have a "vasculitic" phenotype with glomerulonephritis, mononeuritis multiplex and relapses [9, 10]. However, the overall prognosis of ANCA-negative patients remains worse due to a higher frequency of cardiomyopathy [11]. Cardiac involvement is indeed the leading cause of dead [11] in EGPA patients and therefore it must be actively searched, as was the case.

The five-factor score (FFS) is a prognostic tool that consists of five items. Age over 65 years, heart and gastrointestinal tract disease and creatininemia $>150 \mu \mathrm{mol} / \mathrm{L}$ each provide a point. Ear, nose and throat manifestations, however, are associated with a better outcome and its absence is also scored +1 [12]. Patients with an FFS $\geq 1$ are defined as high-risk patients, and treatment is recommended to include not only corticosteroids but also a cytotoxic drug, usually cyclophosphamide. Our patient scored 2 in the FFS and therefore started double treatment as recommended. Prophylaxis for Pneumocystis jiroveci pneumonia with co-trimoxazole ( $800 \mathrm{mg}$ thrice weekly) was also started.

Eosinophil-associated diseases are not always expected and therefore are frequently unrecognized but, in the presence of eosinophilia, the possibility of end-organ damage caused by eosinophils must be considered. The different causes for eosinophilia must then be thoroughly investigated, since they differ in treatment and prognosis. In the case of EGPA, prompt recognition and treatment is imperative to ablate vasculitis tissue damage and improve outcome. Therefore, physicians must be aware of this rare entity and its differential diagnosis.

\section{Grant Support}

None.

\section{References}

1. Rothenberg ME, Hogan SP. The eosinophil. Annu Rev Immunol. 2006;24:147-174.

2. Blanchard C, Rothenberg ME. Biology of the eosinophil. Adv Immunol. 2009;101:81-121.

3. Brito-Babapulle F. The eosinophilias, including the idiopathic hypereosinophilic syndrome. $\mathrm{Br} \mathrm{J}$ Haematol. 2003;121(2):203-223.

4. Pagnoux C, Groh M. Optimal therapy and prospects for new medicines in eosinophilic granulomatosis with polyangiitis (Churg-Strauss syndrome). Expert Rev Clin Immunol. 2016;12(10):1059-1067.

5. Pagnoux C. Churg-Strauss syndrome: evolving concepts. Discov Med. 2010;9(46):243-252.

6. Comarmond C, Pagnoux C, Khellaf M, Cordier JF, Hamidou M, Viallard JF, Maurier F, et al. Eosinophilic granulomatosis with polyangiitis (Churg-Strauss): clinical characteristics and long-term followup of the 383 patients enrolled in the French Vasculitis Study Group cohort. Arthritis Rheum. 2013;65(1):270-281.

7. Moosig F, Bremer JP, Hellmich B, Holle JU, Holl-Ulrich $\mathrm{K}$, Laudien $\mathrm{M}$, Matthis $\mathrm{C}$, et al. A vasculitis centre based management strategy leads to improved outcome in eosinophilic granulomatosis and polyangiitis (ChurgStrauss, EGPA): monocentric experiences in 150 patients. Ann Rheum Dis. 2013;72(6):1011-1017.

8. Masi AT, Hunder GG, Lie JT, Michel BA, Bloch DA, Arend WP, Calabrese LH, et al. The American College of Rheumatology 1990 criteria for the classification of Churg-Strauss syndrome (allergic granulomatosis and angiitis). Arthritis Rheum. 1990;33(8):1094-1100.

9. Sinico RA, Di Toma L, Maggiore U, Bottero P, Radice A, Tosoni C, Grasselli C, et al. Prevalence and clinical significance of antineutrophil cytoplasmic antibodies in ChurgStrauss syndrome. Arthritis Rheum. 2005;52(9):29262935. 
10. Sable-Fourtassou R, Cohen P, Mahr A, Pagnoux C, Mouthon L, Jayne D, Blockmans D, et al. Antineutrophil cytoplasmic antibodies and the Churg-Strauss syndrome. Ann Intern Med. 2005;143(9):632-638.

11. Samson M, Puechal X, Devilliers H, Ribi C, Cohen P, Stern M, Pagnoux C, et al. Long-term outcomes of 118 patients with eosinophilic granulomatosis with polyangii- tis (Churg-Strauss syndrome) enrolled in two prospective trials. J Autoimmun. 2013;43:60-69.

12. Guillevin L, Pagnoux C, Seror R, Mahr A, Mouthon L, Le Toumelin P. The Five-Factor Score revisited: assessment of prognoses of systemic necrotizing vasculitides based on the French Vasculitis Study Group (FVSG) cohort. Medicine (Baltimore). 2011;90(1):19-27. 